\begin{tabular}{|l|l|l|l|}
\hline Eiszeitalter und Gegenroart & Band 12 & Seite 5-8 & Ohringen/Württ. 15. Januar 1962 \\
\hline
\end{tabular}

\title{
Eine Karte des quartären und tertiären Vulkanismus in Europa ${ }^{1}$ )
}

\author{
Von Martin Schwarzbach, Köln \\ Mit 1 Kartenbeilage
}

\begin{abstract}
$\mathrm{Z} u s$ a m m e $\mathrm{f}$ a s $\mathrm{u} \mathrm{ng}$. Die jungen (quartären und tertiären) Vulkangebiete Europas werden auf einer Karte dargestellt und dazu einige allgemeine Bemerkungen über zeitliche Verteilung, petrographische und tektonische Beziehungen des jungen Vulkanismus gemacht.

A bstract. There is given a map of the young (Quaternary and Tertiary) volcanoes in Europe. Some remarks on the chronological, petrographical and tectonic relations of the young
\end{abstract} volcanism.

Der Vulkanismus ist ein Stiefkind der Quartärgeologen. In den großen einschlägigen Lehrbüchern der Eiszeitforschung werden Vulkane allenfalls ganz nebenbei behandelt und meist nur im Zusammenhang mit anderen geologischen Erscheinungen erwähnt.

Immerhin besteht jedoch eine ganze Reihe von Verbindungen: Der junge Vulkanismus ist nicht nur ganz allgemein ein integrierender Bestandteil der Gesamt-Geologie des Eiszeitalters; er hilft darüber hinaus der Quartärstratigraphie und -chronologie (so durch die "Tephrochronologie“ in Patagonien, Island, der Eifel), lieferte Material für den Steinzeitmenschen (Obsidian), gibt Klimazeugen (Windrichtungen bei Aschen-Eruptionen) und ist vielfach als Eiszeit-Ursache diskutiert worden. Schließlich haben auch die jungen vulkanischen Gesteine eine große Rolle in den modernen Untersuchungen über Paläomagnetismus übernommen, nicht nur bei der Bestimmung der Pol-Lagen in quartärer Zeit, sondern auch dadurch, daß die mehrfachen Umkehrungen des magnetischen Feldes um $180^{\circ}$ offenbar chronologisch verwertet werden können; man kann sich vorstellen, daß solche Beobachtungen sogar bei der Frage nach der Abgrenzung des Quartärs gegen das Tertiär Bedeutung gewinnen.

In der beiliegenden $\mathrm{K}$ a $\mathrm{r}$ te ist versucht worden, die räumliche Verbreitung des effusiven jungen Vulkanismus darzustellen. Dabei wurde außer dem Quartär auch das Tertiär mit behandelt, denn beide hängen eng zusammen; in vielen Fällen ist außerdem eine ganz genaue stratigraphische Einordnung der vulkanischen Vorgänge gar nicht möglich. Allerdings besteht im allgemeinen ein scharfer morphologischer Schnitt zwischen beiden Zeitabschnitten, denn Krater und ähnliche morphologische Einzelheiten sind nur an quartären Vulkanen überliefert; die tertiären sind viel stärker abgetragen.

$\mathrm{Zu}$ der Karte sollen nur wenige allgemeine Bemerkungen gemacht werden.

1. Zeitliche Verteilung des jungen Vulkanismus

Nach dem zeitlichen Auftreten lassen sich drei Hauptgruppen unterscheiden:

a) Gebiete mit rein tertiärem Vulkanismus und höchstens ganz vereinzelten quartären Ausbrïchen,

b) Gebiete mit rein quartärem oder ganz überwiegend quartärem Vulkanismus (darin als Untergruppe die Gebiete mit „tätigen“ Vulkanen),

c) Gebiete, in denen tertiärer und quartärer Vulkanismus eine bedeutende Rolle spielen.

Zur Gruppe a) gehören die südöstliche Balkanhalbinsel (mit nördlicher Ägäis), das innerkarpathische Vulkangebiet, das ost- und südalpine Vulkangebiet, die meisten

1) Die Karte wurde erstmalig in einem öffentlichen Vortrag über „Vulkane des Eiszeitalters in Europa“ anläßlich der Hauptversammlung der Deutschen Quartärvereinigung in Karlsruhe am 9. Juni 1960 gezeigt. 
Vulkangebirge Mittel-Europas (mit Ausnahme der Eifel), Schonen und die südliche ThuleProvinz (Schottland-Irland-Färöer).

Zur Grup pe b) sind vor allem zu rechnen: südliche Agäis, die Apennin-Halbinsel mit Nordost-Sizilien und den zirkumsizilischen Vulkaninseln, Olot. Auch Ciudad Real könnte man noch dazu rechnen.

In die Gruppe c) muß man vor allem stellen: Sardinien, Auvergne, Eifel und Island.

Es zeigt sich somit, daß nur im Mittelmeerraum noch im Laufe des Quartärs (z. T. im jüngsten Tertiär) neue Vulkangebiete entstehen; alle anderen sind älter. Der Schwerpunkt vulkanischer Tätigkeit liegt während des Tertiärs im mittleren und südöstlichen Europa, ferner in der Umrandung des westlichen Mittelmeeres und schließlich in der Thule-Provinz. Nur in kleinem Umfange setzt sich in diesem Raum die Tätigkeit in festländischen Einzelgebieten (Auvergne, Eifel, Sardinien) ins Quartär hinein fort, dagegen in großem Umfange in Island. Im übrigen verschiebt sich im Quartär der Schwerpunkt nach dem mittleren und östlichen Mittelmeerraum.

\section{Petrographische Zusammenhänge}

Der junge Vulkanismus hat vorwiegend basische Gesteine geliefert. Doch gibt es auch in ausgesprochenen Basaltgebieten, z. B. in Island oder der West-Eifel, gelegentlich saure Ergüsse, und außerdem auch größere Gebiete mit reichlicher Förderung von liparitischer oder trachytischer Lava. Die Art der vulkanischen Tätigkeit hängt weitgehend davon $a b$; die trachytischen Staukuppen vom Typ des Puy de Dôme sind Beispiele für saure, sehr zähflüssige Laven, die isländischen Schildvulkane für dünnflüssiges, basaltisches Magma.

Von größerem Interesse ist aber die Zugehörigkeit der Magmen zu bestimmten vulkanischen Provinzen, wenn auch die Laven keineswegs immer scharf der pazifischen, atlantischen oder mediterranen Sippe im Sinne F. BECKE's und P. NigGli's zuzuordnen sind.

Für die Hauptgebiete ergibt sich (vgl. zuletzt A. Ritrmann 1960):

I s 1 a n d. Pazifisch-atlantisch.

I be $\mathrm{r}$ is che $\mathrm{H}$ a $1 \mathrm{~b}$ in se 1 . Im Meseta-Gebiet atlantisch, im betischen Gebiet pazifisch mit mediterranem Einschlag.

A uvergne. Atlandisch bis pazifisch.

Eif el (und übriges Mitteleuropa). Ganz überwiegend atlantisch.

In nen rand der Karpathen. Pazifisch.

Ostliches und südliches Alpenvorland. Pazifisch-mediterran.

Tosk a n a. Pazifisch-mediterran.

Mittel-Italien. Mediterran.

Liparische Inse $1 \mathrm{n}$. Pazifisch-mediterran.

A t $\mathrm{n}$ a. Atlantisch.

$\dddot{A} \mathrm{~g}$ ä is. Pazifisch, z. T. atlantisch oder mediterran.

\section{Tektonische Beziehungen}

Die engen Beziehungen zwischen Vulkanismus und Tektonik, die man in vielen Teilen der Erde erkannt hat, treten auch in Europa heraus.

Zunächst fällt auf, daß von den großen tektonischen Einheiten unseres Kontinents Ur- und Paläo-Europa (d. h. Russische Tafel, Skandinavien, der größte Teil der Britischen Inseln) völlig frei von quartären Eruptiven sind, und auch tertiäre Vulkane treten dort nur vereinzelt auf (Schonen, Nord-Irland, Innere Hebriden). Aber die Vorkommen in Schottland und Irland gehören auch gar nicht mehr zum eigentlichen festländisch-europäischen Vulkanismus, sondern bereits zu der im wesentlichen außereuro- 
päischen Thule-Provinz. Es besteht also tatsächlich ein fast vollständiger scharfer Schnitt zwischen Ur- und Paläo-Europa einerseits, Neo- und Meso-Europa andererseits.

Dabei ist Neo-E u ropa, d.h. das Gebiet der alpidischen Faltungen, das Hauptgebiet des jungen Vulkanismus, aber die tektonischen Kräfte, die hier den Magmenaufstieg aktivierten, entfalteten sich auch im angrenzenden Meso-Europa, teilweise sogar sehr kräftig, und in einem Fall, nämlich in Schonen, sogar in dem weiter entfernten Ur-Europa.

Wir können also unterscheiden:

a) Vulkanismus in Verbindung mit den alpidischen Faltungen (der gesamte festländische junge Vulkanismus einschließlich des Mittelmeerraums),

b) Vulkanismus der Thule-Provinz (Island, Färöer, Britische Inseln).

4. Der junge Vulkanismus im Bereich der alpinen Faltungen

Innerhalb von Neo-Europa sind freilich die eigentlichen Faltengebirge und vor allem deren zentrale Teile (d.h. die tektonisch am stärksten betroffenen Teile) vielfach überhaupt frei von Vulkanen. Das gilt besonders für die Alpen selbst, aber auch für Schweizer Jura, Pyrenäen, Dinariden, Karpathen und Balkan. Nur vereinzelt finden sich Eruptivzentren in zentraler Lage. Offenbar ist der Zusammenschub der Schichten meist so stark gewesen, daß dem Magma der Weg nach oben versperrt war. Wohl aber konnten sich Plutone bilden, die inzwischen $\mathrm{z}$. T. von der Abtragung freigelegt worden sind (Granite von Adamello usw.).

Vorwiegend liegen die Gebiete mit jungem Vulkanismus vielmehr a $\mathrm{m} R$ a $\mathrm{nde} d$ e $\mathrm{r}$ F a l teng ebirge, ja, z. T. ziemlich weit entfernt. Dabei kommen sowohl die zwischen den Faltenschlingen gelegenen "Zwischengebirge" als auch das nach außen hin folgende Vorland der Geosynklinalen in Frage. So begleiten den Innenrand des Karpathenbogens die Vulkangebirge der nördlichen und östlichen Pannonischen Masse von Kremnitz bis nach Kronstadt; der südostbalkanische, ägäische und kleinasiatische Vulkanismus ist an die Ränder von Rhodopen- und Kykladen-Masse geknüpt. Das z. T. versunkene Tyrrhenische Massiv wird von den apenninischen Vulkanen umgürtet; Ätna und Liparische Inseln erheben sich am Rand der peloritanisch-calabrischen Masse. Sardinien gehört gänzlich zu einem alten Massiv. Die Vulkane der spanischen Südost-Küste bezeichnen wie die der algerisch-tunesischen Küste die Grenze zwischen jungen Faltenzügen und dem westlichen Mittelmeerbecken, das wohl einer versunkenen „algero-betischen Masse“ entspricht.

Dem „mesoeuropäischen“, außerhalb der jungen Falten gelegenen Vorland gehören als wichtigste die Vulkane Zentral-Frankreichs und Mittel-Europas an.

In all diesen randlichen Gebieten hat sich das tektonische Geschehen im Tertiär und Quartär weitgehend in einer tiefgründigen Zerspaltung und Schollentektonik geäußert.


Meeresspiegel. Zerrungsvorgänge öffneten dem Magma den Weg nach oben.

Hier größere Gesetzmäßigkeiten zu erkennen, ist schwierig. Für den mitteleuropäischen Raum hat es $\mathrm{H}$. CLoos versucht, der von der großräumigen Aufbeulung eines „Rheinischen Schildes" und dessen Zerspaltung ausging. Ebenso ist sicher, daß Vulkankegel reihenförmig angeordnet sein können, d.h. auf lang durchlaufenden Spalten aufsitzen (z. B. Chaîne des Puys), oder Vulkangebiete wenigstens ausgeprägte Längserstreckung zeigen. Doch meist lassen sich auch solche kleinregionalen Beziehungen nicht eindeutig erkennen. KNETSCH hat vermutet, daß der Vulkanismus deswegen nicht immer ,gesetzmäßig zur erdoberflächlich sichtbaren Tektonik angeordnet erscheint“, weil er tektonische Verhältnisse tieferer Krustenstockwerke widerspiegelt.

Betrachten wir die Zugehörigkeit der vulkanischen Gesteine zu den MagmenProvinzen, so lassen sich in diesem tektonischen Rahmen immerhin einige Überein- 
stimmungen finden. Pazifische Magmen sind auf die eigentlichen Faltengürtel (im weiteren Sinne, d. h. einschließlich der z. T. kräftig mit „verarbeiteten “ Zwischengebirge) beschränkt (Karpathen, Ägäis). Aber es gibt auch da vielfach Übergänge zu mediterranen und atlantischen Sippen. Der Apennin-Raum ist das klassische Gebiet der kalireichen mediterranen Sippe (in abgeschwächtem Maße zeigt auch der Kaiserstuhl im OberrheintalGraben mediterranen Charakter). Das nördliche Vorland gehört im wesentlichen der atlantischen Provinz an.

$\mathrm{Ze}$ itlich gesehen, beginnt der mit der alpidischen Orogenese verknüpfte junge Vulkanismus im Vorland, aber auch in manchen $Z$ wischengebirgen. In einigen von diesen Gebieten setzt er sich bis ins Quartär hin fort. Im apenninischen und östlichen Mittelmeerraum setzt er erst im Quartär richtig ein, ja, nur hier besteht er bis zur Jetztzeit fort. Man wird das als Zeichen dafür werten können, daß das östliche Mittelmeer jünger als das westliche ist, und dem würde auch die größere Seismizität im Osten entsprechen.

\section{Der junge Vulkanismus des nordatlantischen Raumes}

Völlig anders ist die tektonische Stellung der Vulkangebiete im nordatlantischen Raum, d. h. im Rahmen unserer Betrachtungen in der europäischen "Thule-Provinz “. Diese zeigt überhaupt eine Reihe von Eigentümlichkeiten gegenüber den eben behandelten Vulkangebieten des alpidischen Europas:

1. Basalte herrschen weitaus vor.

2. Plateau-Basalte spielen eine große Rolle.

3. Der Vulkanismus beginnt sehr zeitig im Tertiär (vielleicht z. T. in der Oberkreide) und setzt sich, wenn auch möglicherweise mit längeren Ruhepausen, bis in die Jetztzeit kräftig fort.

4. Die Vulkane sind ohne Beziehung zu jungen Faltengebirgen.

5. Die Eruptionen sind besonders ausgeprägt an Spalten gebunden.

Wir müssen annehmen, daß hier sehr tiefe Spalten bis in das Substrat der Kruste reichen und basaltischen Magmen immer wieder den Aufstieg ermöglichen. Die Spalten stehen nicht zu orogenetischen Vorgängen in Beziehung, wie im kontinentalen Europa, sondern vielleicht unmittelbar zu gewaltigen langsamen Strömungsbewegungen im tieferen Untergrund der Erde; diese führen zu Zerrungsvorgängen und Magmenaufstieg, denen möglicherweise auch die Mittelaltlantische Schwelle ihr Dasein verdankt.

\section{Wichtiges Schriftum}

Burri, C. \& Niggli, P.: Die jungen Eruptivgesteine des mediterranen Orogens. 2 Bde. - Publ. Vulkaninst. I. Friedländer, 3, $654+206$ S., Zürich 1945 u. 1949.

Cloos, H.: Hebung - Spaltung - Vulkanismus. - Geol. Rdsch. 30, S. 405-525, Bonn 1939.

GlangEAud, L.: Les éruptions tertiaires nord-africaines, leurs relations avec la tectonique méditerranéenne. - Congr. Géol. Int. 1952 Alger, C. R., XVII, S. 71-101, Alger 1954.

KNEtsch, G.: Die tektonische Stellung des jungen Vulkanismus in Mitteleuropa. - Abh. Braunschw. Wiss. Ges., 2, S. 92-103, Braunschweig 1950.

Kraus, E.: Die Entwicklungsgeschichte der Kontinente und Ozeane. - 285 S., Berlin 1959.

Ritrmann, A.: Vulkane und ihre Tätigkeit. 2. Aufl. - 336 S., Stuttgart 1960.

SAPPER, K.: Vulkankunde. - 424 S., Stuttgart 1927.

SEIDLITZ, W. v.: Diskordanz und Orogenese der Gebirge am Mittelmeer. - 651 S., Berlin 1931.

Stille, H.: Der geotektonische Werdegang der Karpathen. - Beih. Geol. Jb. 8, 239 S., Hannover 1953.

Termier, H. \& G.: L'évolution de la lithosphère. II. Orogénèse. 2 Bde. - 940 S., Paris 1956 u. 1957.

WolfF, F. v.: Der Vulkanismus. I u. II. - 711 + 1111 S., Stuttgart 1914-1931.

Manuskr. eingeg. 9. 6. 1961.

Anschrift des Verf.: Prof. Dr. M. Schwarzbach, Geologisches Institut der Universität, Köln, Zülpicher Straße 47. 